\title{
De novo assembly and transcriptome characterization: Novel insights into the mechanisms of primary ovarian cancer in Microtus fortis
}

\author{
QI HU ${ }^{1-3^{*}}$, MINGYUE GAO ${ }^{3 *}$, DU ZHANG ${ }^{3}$, BINGFENG LENG $^{3}$, JUNWEN WANG $^{3}$, \\ QIAN LIU ${ }^{1,2}$, SHUANGYAN HE ${ }^{1,2}$, WENLING ZHI ${ }^{1,2}$ and ZHIJUN ZHOU ${ }^{1,2}$ \\ ${ }^{1}$ Department of Laboratory Animal Science, Xiangya Medical College; ${ }^{2}$ Hunan Key Laboratory of \\ Animal Models for Human Diseases, Central South University, Changsha, Hunan 410013; \\ ${ }^{3}$ Department of Bioinformatics Center, NEOMICS Institute, Shenzhen, Guangdong 518118, P.R. China
}

Received April 6, 2021; Accepted September 2, 2021

DOI: $10.3892 / \mathrm{mmr} .2021 .12580$

\begin{abstract}
The natural incidence of primary epithelial ovarian cancer (OVC) in adult female voles of some established strains of Microtus fortis is relatively high. M. fortis OVC has some pathological similarities to human epithelial OVC, therefore $M$. fortis represents the latest and most valuable animal model for studying human OVC. The lack of available genetic information for $M$. fortis limits the use of common immunological methods; thus, high-throughput sequencing technologies have been used to reveal the mechanisms of primary OVC in $M$. fortis. The individuals with cancer were diagnosed using histopathologic hematoxylin and eosin staining. The present study used RNA-sequencing (RNA-seq) technology to establish a de novo assembly of the $M$. fortis transcriptome produced 339,830 unigenes by the short reads assembly program Trinity. Comparisons were made between OVC and healthy ovarian tissue (OV) and between fallopian tube cancer (FTC) and healthy fallopian tube (FT) tissues using RNA-seq analysis. A total of 3,434 differentially expressed genes (DEGs) were identified in OVC tissue compared with OV tissue using RNA-Seq by Expectation-Maximization software, including 1,950 significantly upregulated and 1,484 significantly downregulated genes. There were 2,817 DEGs identified in the FTC tissues compared with the FT tissue, including 1,762 significantly upregulated and 1,055 significantly downregulated genes.
\end{abstract}

Correspondence to: Professor Zhijun Zhou, Department of Laboratory Animal Science, Xiangya Medical College, Central South University, 172 Tongzipo Road, Changsha, Hunan 410013, P.R. China

E-mail: zhouzhijun@csu.edu.cn

${ }^{*}$ Contributed equally

Key words: Microtus fortis, primary ovarian cancer, epithelial ovarian cancer, RNA-sequencing
Pathway enrichment analysis revealed that upregulated transcripts in the OVC vs. OV groups were involved in cell growth and proliferation-associated pathways, whereas the downregulated DEGS in the OVC vs. OV groups were enriched in steroid biosynthesis-related pathways. Furthermore, the tumor suppressor gene, p53, was downregulated in the FTC and OVC compared with the FT and OV groups, respectively; whereas, genes that promoted cell migration, such as Ras-related protein Rap- $1 \mathrm{~b}$, Ras homolog family member A and RAC1, were upregulated. In summary, to the best of our knowledge, the present study characterized the M.fortis de novo transcriptome of $\mathrm{OV}$ and FT tissues and to perform RNA-seq quantification to analyze the differences in healthy and cancerous OV and FT tissues. These results identified pathways that differed between cancerous and healthy M.fortis tissues. Analysis of these pathways may help to reveal the pathogenesis of primary OVC in M. fortis in future work.

\section{Introduction}

For worldwide, ovarian cancer (OVC) is the one of top 10 most common type of women cancer, while as the same common as in China (1). Early diagnosis of OVC is difficult to achieve, as the main symptoms are not OVC-specific (2). Therefore, most patients with OVC are diagnosed with metastatic cancer, with a 5-year survival rate of $29 \%(3,4)$. OVC can metastasize through two different mechanisms (5-7): i) The primary tumor can expand and directly infiltrate adjacent organs, including the bladder and colon; or ii) cancer cells can become isolated from the primary tumor and spread to the peritoneal cavity, an event usually associated with the formation of ascites. OVC is easy to metastasize, and patients with OVC often present with advanced pelvic disease (8), including expansion of the cancer into the uterus, fallopian tubes and ovaries (9). In vivo animal models are essential tools for cancer research (10); they have enabled the identification of carcinogens, the development of cancer therapies (11) and high-throughput drug screening (12), and improved our understanding of the molecular mechanisms of tumor growth and metastasis (13). 
OVC models must be established to enhance our current understanding of the biological characteristics of OVC and to develop novel and improved therapeutics (14). Mice are often used as in vivo models of human disease owing to high similarities of gene homologous. Previous experiments on Microtus fortis found that in some established strains the natural incidence of OVC in adult female voles is markedly higher than that exhibited in other animals in the Department of Laboratory Animal Science, Xiangya Medical College, Central South University (15). Through clinical and pathological observations, it is demonstrated that $M$. fortis OVC shares a number of similarities with human $\operatorname{OVC}(16,17)$. To elucidate the underlying mechanisms of human OVC pathogenesis, the pathological characteristics and histological classification of spontaneous epithelial OVC in $M$. fortis can be used.

Unlike mouse models, less available biological information is the limitation of $M$. fortis as a novel experimental animal model. It is therefore challenging to study the underlying mechanisms of primary OVC in $M$. fortis using techniques such as flow cytometry, western blotting and microarrays (18). De novo RNA-seq technology is useful to analyze gene expression and identify novel genes (19). Therefore, the present study aimed to sequence and compare the $M$. fortis transcriptomes in OVC and healthy ovarian (OV) tissues and between fallopian tube cancer (FTC) and healthy fallopian tube (FT) tissues, using Illumina sequencing platform. The results demonstrated the suitability of short-read sequencing for de novo transcriptome assembly and for the annotation of genes expressed in a eukaryote with no previous genome information. The present study also identified differentially expressed genes (DEGs) between FTC and FT and between OVC and OV tissue samples. Gene Ontology (GO) and Kyoto Encyclopedia of Genes and Genomes (KEGG) enrichment analyses were performed to determine the functional relationships of the identified DEGs. The results demonstrated that cysteine-rich angiogenic inducer 61 (CYR61), Ras-related protein Rap-1b (RAP1B), Ras homolog family member A (RHOA), RAC1 and BTG antiproliferation factor (BTG) 3 may serve an important role in the pathogenesis of primary OVC in M. fortis.

\section{Materials and methods}

Animals and sample preparation. M. fortis, originating from the Dongting Lake area (Yueyang, China) were bred in the laboratory. All animals were raised in cages maintained in a 12-h light/dark cycle at a temperature of $22 \pm 1.5^{\circ} \mathrm{C}$ and $50 \pm 5 \%$ relative humidity. Animals were fed with standard formula feed for $M$. fortis (sterilized by $\gamma$-radiation from a Cobalt-60 source) and sterile water. The housing environment was controlled by an automatic heating and ventilation device, and the bedding materials were sterilized in advance. During the pedigree breeding process, $M$. fortis were found to have OVC or FTC with an unstable ratio $(\sim 4 \%)$ as the number of animals with OVC or FTC changed in each generation. In the present study, a total of $110 \mathrm{M}$. fortis females were produced from the breeding process and four were obtained with cancer. The average age of the animals discovered with cancer was 1 year while the average lifetime is 3-4 years. The animals with cancer were observed to have ascites and sluggish movements (maximum weight gain $100 \mathrm{~g}$; Fig. S1). M. fortis were euthanized by $4 \%$ isoflurane overdose, following an induction with $4 \%$ isoflurane, using an anesthesia machine (ABM model; Shanghai Yuyan Instruments Co., Ltd.); animals underwent prolonged exposure to $4 \%$ isoflurane to ensure death ( $>3 \mathrm{~min}$ ). Death was verified when there was no breathing for $>2 \mathrm{~min}$ and no blink reflex $(20,21)$. All animal experiments were performed according to the Laboratory Animal Guidelines for Euthanasia (T/CALAS 31-2017; Chinese Association for Laboratory Animal Sciences; http://www.calas.org.cn/index.php? $\mathrm{m}=$ content $\& \mathrm{c}=\mathrm{index} \& \mathrm{a}=\mathrm{s}$ how\&catid=19\&id=1007) within the recommendations of the Laboratory of Animal Welfare \& Ethics Committee of China. The protocol was approved by the Laboratory Animal Welfare and Ethics Committee of Central South University (Changsha, China; approval no. 2018sydw0236). Cancerous and healthy tissues were collected from four animals with OVC and two healthy animals. Samples were stored in an ultra-low temperature freezer $\left(-80^{\circ} \mathrm{C}\right)$.

Pathological observations. For individuals with ascites, cancer was diagnosed using histopathologic hematoxylin and eosin (H\&E) staining (Fig. S1). The fresh tissue was fixed with a FAA fixative solution (catalogue no. G1103-500ML; ServiceBio, Inc.) for $>24 \mathrm{~h}$ and then dehydrated by gradient alcohol in the dehydrator (Histocentre 3; Thermo Fisher Scientific, Inc.) and soaked in paraffin wax. The paraffin wax-soaked tissues were cooled on a $-20^{\circ} \mathrm{C}$ freezing table and then sliced to of $3 \mu \mathrm{m}$ using a rotary microtome (Finesse E+; Thermo Fisher Scientific, Inc.). The slices were floated in $40^{\circ} \mathrm{C}$ warm water to flatten the tissue and then baked in an oven at $60^{\circ} \mathrm{C} 1 \mathrm{~h}$. Slices were dried and then stored at room temperature. The sections were incubated with xylene for $40 \mathrm{~min}$, absolute ethanol for $10 \mathrm{~min}$ and $75 \%$ ethanol for $5 \mathrm{~min}$, then washed with distilled water, stained with hematoxylin for $5 \mathrm{~min}$ and then differentiated with $1 \%$ hydrochloric acid and alcohol for $10 \mathrm{sec}$ (all at room temperature). The slices were washed with distilled water, then returned to blue for $20 \mathrm{~min}$ at room temperature with $0.6 \%$ ammonia water and rinsed with running distilled water. Slices were separately dehydrated $5 \mathrm{~min}$ at room temperature with 85 and $95 \%$ alcohol in succession and then stained in eosin solution for $5 \mathrm{~min}$. The slices were successively put into anhydrous ethanol $15 \mathrm{~min}$ and xylene $15 \mathrm{~min}$ at room temperature until they were transparent and were then sealed with neutral gum. All the above incubation, dewaxing and dyeing processes were performed at room temperature. The morphological changes in the tissues were observed under an optical light microscope for 22 fields (BX43; Olympus Corp.).

cDNA library construction and Illumina sequencing. Total RNA of $12 \mathrm{M}$. fortis ovary (two healthy and four cancerous) and fallopian tube (two healthy and four cancerous) tissue samples was extracted using the RNeasy mini kit (Qiagen $\mathrm{GmbH})$. RNA degradation and contamination were monitored on $1 \%$ agarose gels. RNA purity was checked using a NanoPhotometer ${ }^{\circledR}$ spectrophotometer (Implen, Inc.). RNA concentration was measured using a Qubit ${ }^{\circledR}$ RNA Assay kit and a Qubit ${ }^{\circledR}$ 2.0 Fluorometer (Thermo Fisher Scientific, Inc.). RNA integrity was assessed using the RNA Nano 6000 Assay kit and the Agilent Bioanalyzer 2100 system (Agilent 
Technologies, Inc.). A total amount of $1.5 \mu \mathrm{g}$ RNA per sample was used as input material. Sequencing libraries were generated using the NEBNext ${ }^{\circledR}$ Ultra $^{\mathrm{TM}}$ RNA Library Prep kit for Illumina ${ }^{\circledR}$ (catalogue no. E7530L; New England BioLabs, Inc.) according to the manufacturer's protocol. Briefly, mRNA was purified from total RNA using poly-T oligo-attached magnetic beads. mRNA was fragmented when mixed with fragmentation buffer. cDNA was synthesized using the mRNA fragments as templates. To select cDNA fragments of the right length, the library fragments were purified using the AMPure XP system (Beckman Coulter, Inc.). Subsequently, $3 \mu$ USER enzyme (New England BioLabs, Inc.) was incubated with the size-selected, adaptor-ligated cDNA at $37^{\circ} \mathrm{C}$ for $15 \mathrm{~min}$ followed by $5 \mathrm{~min}$ at $95^{\circ} \mathrm{C}$ prior to PCR. PCR was performed using Phusion High-Fidelity DNA polymerase (catalogue no. M0530L; NEB, Inc.), universal PCR primers and PCR index (Table SI), following initial denaturation $\left(94^{\circ} \mathrm{C}\right.$, $1 \mathrm{~min}) ; 12$ of cycles of denaturation $\left(94^{\circ} \mathrm{C}, 30 \mathrm{sec}\right)$, annealing $\left(58^{\circ} \mathrm{C}, 30 \mathrm{sec}\right)$ and elongation $\left(72^{\circ} \mathrm{C}, 30 \mathrm{sec}\right)$, and final extension $\left(72^{\circ} \mathrm{C}, 2 \mathrm{~min}\right)$. Finally, products were purified (AMPure XP system) and library quality was assessed using the Agilent Bioanalyzer 2100 system. From these libraries (3-7 ng/ $\mu 1$, measured by Qubit), 150 bp paired-end and strand-specific sequence reads were produced using the Illumina HiSeq $X$ Ten (Illumina, Inc.) at E-GENE Co., Ltd.

Quality control and de novo assembly for transcriptome analysis. The raw reads were initially processed using Illumina sequencing, aforementioned. Quality control of raw reads was performed using FastQC (version 0.11.5; www.bioinformatics. babraham.ac.uk/projects/fastqc), and clean reads were obtained following the removal of low-quality reads with Trimmomatic (version 0.36; https://github.com/usadellab/Trimmomatic) software (22). Subsequently, de novo transcriptome assembly was performed using the short reads assembly program, Trinity (version 2.3.2, https://github.com/trinityrnaseq/trinityrnaseq/wiki) (23). The final sequences obtained after assembly were termed unigenes. Clean reads were mapped to the de novo assembly transcriptome reference sequences of $M$. fortis using Bowtie2 (version 2.4.1, http://bowtie-bio.sourceforge. net/bowtie2/index.shtml). The expression levels of unigenes were calculated using the RSEM algorithm. The hierarchical cluster analysis was performed via function hclust in vegan $\mathrm{R}$ package (version 2.51. http://www.R-project.org/) (24).

Functional annotation. TransDecoder (version 2.0.1; github. com/TransDecoder/TransDecoder/wiki) was used to identify open reading frames (ORFs) and to predict potential coding sequences in the assembled unigenes. After ORFs were extracted from the assembly, the Trinotate (version 2.0.2 github.com/Trinotate/Trinotate) pipeline was used to annotate the $M$. fortis transcript ORF dataset. Both nucleotide transcripts and protein sequences were used to search against the UniProt Knowledgebase (KB)/Swiss-Prot databases (www. uniprot.org), using basic local alignment search tool BLASTx and BLASTp [version 2.2.28+; cut-off level (E-value), 1x10 ${ }^{-5}$ ], respectively. The UniProtKB/Swiss-Prot database is a collection of functional information on proteins, with accurate, consistent and rich annotation. Protein domains were annotated using the Pfam domain database with HMMER (version 3.1, http://www.hmmer.org/) (25). Potential signal peptides were predicted using SignalP (version 4.1) (26). All M. fortis transcriptome annotations were loaded into the SQLite database (https://www.sqlite.org/index.html) and the annotated results were exported to an excel file.

Abundance estimating and screening of DEGs. The expression of each unigene was quantified by RNA-seq quantification analysis using RNA-Seq by Expectation-Maximization (RSEM) software (version 1.2.31, https://github.com/deweylab/RSEM). Unigenes that were expressed at low levels (FPKM in all samples $<1.0$ ) were removed from the database. DEGs between OVC and OV and between FTC and FT tissues were determined using the DESeq2 R package (27) (q-value $<0.001$; $\log 2$ fold-change $>1$ ). GO terms (geneontology.org) were distributed into 'Biological Processes' (BP), 'Cellular Components' (CC) and 'Molecular Functions' (MF). KEGG (www.kegg.jp) is the main public database related to pathways, usually used for enrichment analysis of DEGs. Subsequently, GO term and KEGG pathway enrichment analyses for the DEGs were executed using GOstats (version 2.50.0, http://bioconductor. riken.jp/packages/release/bioc/html/GOstats.html) and GSEABase (version 1.46.0, http://bioconductor.riken.jp/packages/3.0/bioc/html/GSEABase.html) packages with $\mathrm{P}<0.01$ as a threshold, according to guidance from Trinity.

\section{Results}

Illumina sequencing and de novo transcriptome assembly. To obtain an overview of the $M$. fortis gene expression profile, cDNA from four OVC, two OV, four FTC and two FT tissues was generated and sequenced using the Illumina sequencing platform. After removing the adaptor sequences and low-quality reads, 331,138,454 clean paired-end reads were obtained from the 12 sequenced samples (average size, $7.86 \pm 1.06 \mathrm{~Gb}$ ). The average GC content and average Q30 values were 48.67 and $96.72 \%$, respectively (Table SII). The clean reads were assembled using Trinity software. A total of 521,853 contigs and 339,830 unigenes (average length, 874.6 bp; N50, 1,444 bp) were obtained (Table SIII). The read mapping ratio of all samples was $>80 \%$ (Fig. 1A). Density analysis of all unigene expression levels in almost all samples displayed a similar expression pattern except FTC4, while the lower expression level genes of FTC4 were more compared with the other samples (Fig. 1B). After removing low expression (FPKM $<1.0$ in all samples) unigenes, 104,443 unigenes remained. Cluster analysis indicated that there were not only differences in expression between healthy and cancerous tissues, but also between different organs (Fig. 1C).

Functional annotation and classification of assembled transcripts. To annotate the unigenes, sequences of unigenes were searched using BLASTx against the Swiss-Prot protein and Pfam databases with a cut-off E-value of $1 \times 10^{-5}$. A total of 44,511 (42.62\%) genes and 13,949 (13.35\%) unigenes were identified as significant hits using the respective databases (Table SIV). These annotated results demonstrated that the annotated sequences percentages of species, Mus musculus (52.25\%), Homo sapiens (13.84\%) and Rattus norvegicus (13.48\%) were highly homologous with M. fortis. In the 
A

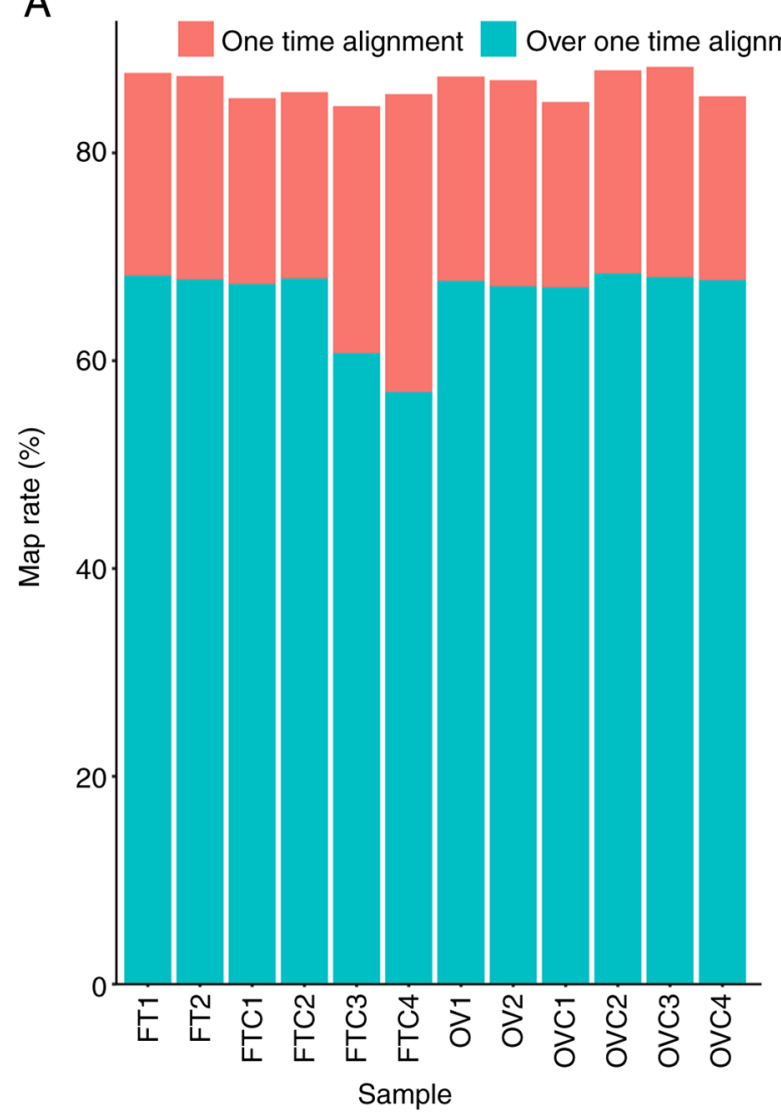

B

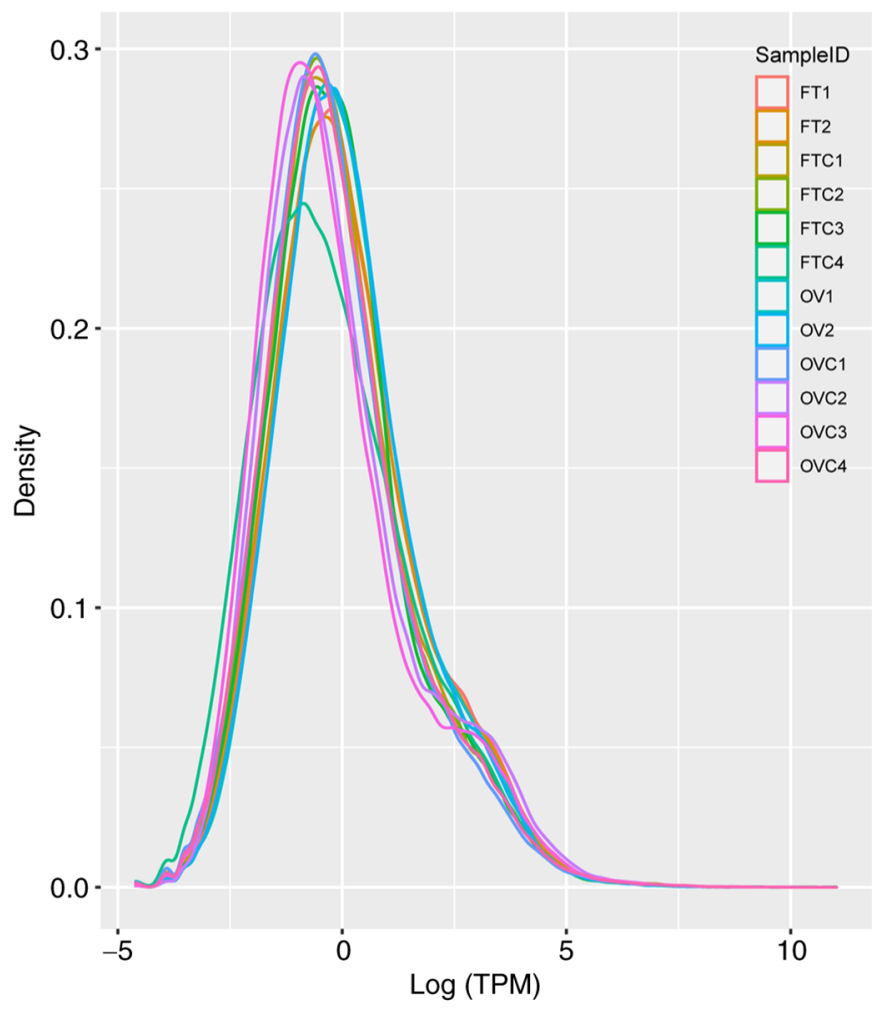

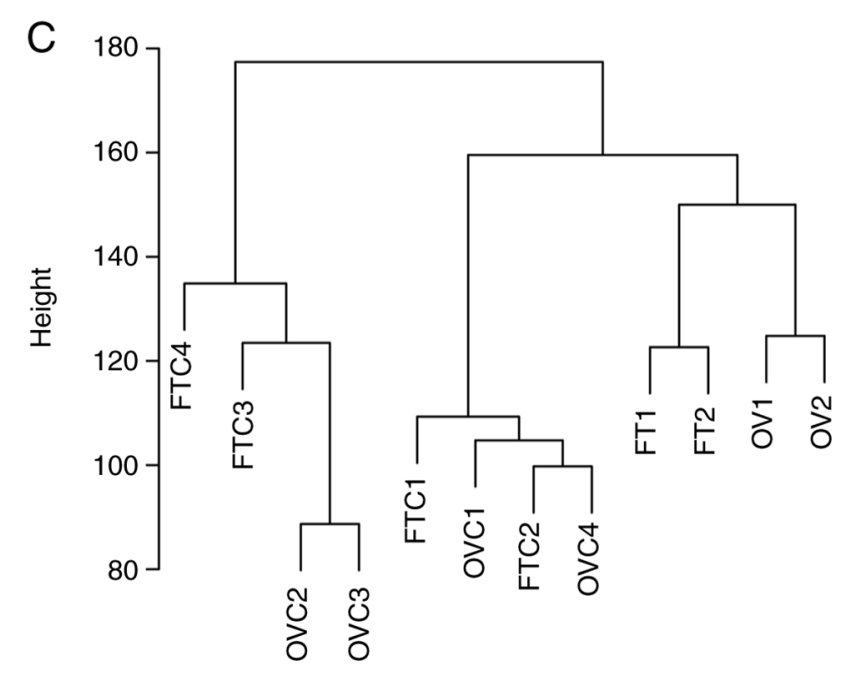

Figure 1. Basic transcriptome and unigenes profiles. (A) Clean reads aligned to de novo assembly transcriptomes. (B) Density plots of unigene expression levels in all samples. (C) Cluster analysis of all samples. FT, healthy fallopian tubes; FTC, fallopian tube cancer; OV, healthy ovarian tissue; OVC, ovarian cancer.

GO database 40,725 unigenes were annotated to $15659 \mathrm{GO}$ number. Among these annotated unigenes, 11,277 (27.69\%) were categorized as cytoplasm belonged to CC terms, 9,017 $(22.14 \%)$ as nucleus belonged to $\mathrm{CC}$, and $8,935(21.94 \%)$ as metal ion binding belonged to MF (Table SV).

In the present study, 21,206 unigenes were mapped to 479 KEGG pathways (Table SVI), including 'chromosome and associated proteins' (2,148 unigenes to 734 koIDs; $10.13 \%$ of sequences), 'membrane trafficking' (2101 unigenes to 547 koIDs; $10.40 \%$ of sequences), 'ubiquitin system' $(1,830$ unigenes to $548 \mathrm{koIDs} ; 8.63 \%$ of sequences), 'exosome' (1,725 unigenes to 508 koIDs; $8.13 \%$ of sequences), 'transcription factors' (1,222 unigenes to 461 koIDs; $5.78 \%$ of sequences), 'transporters' (1107 unigenes to 496 koIDs; $5.22 \%$ of sequences), 'protein kinases' (1042 unigenes to 367 koIDs; $4.91 \%$ of sequences) 'peptidases' (983 unigenes to 397 koIDs; $4.64 \%$ of sequences), 'Mitochondrial biogenesis' (982 unigenes to 230 koIDs; $4.63 \%$ of sequences) and 'pathways in cancer' (922 unigenes to 346 koIDs; $4.35 \%$ of sequences). Out of the 479 KEGG pathways, 16 were identified as being involved in cancer, including, 'pathways in cancer' (922 unigenes to 346 koIDs; $4.35 \%$ ), 'proteoglycans in cancer' (426 unigenes to 143 koIDs; $2.01 \%$ ), 'microRNAs in cancer' (297 unigenes to 115 koIDs; $1.40 \%$ ), 'gastric cancer' (247 unigenes to 90 koIDs; 

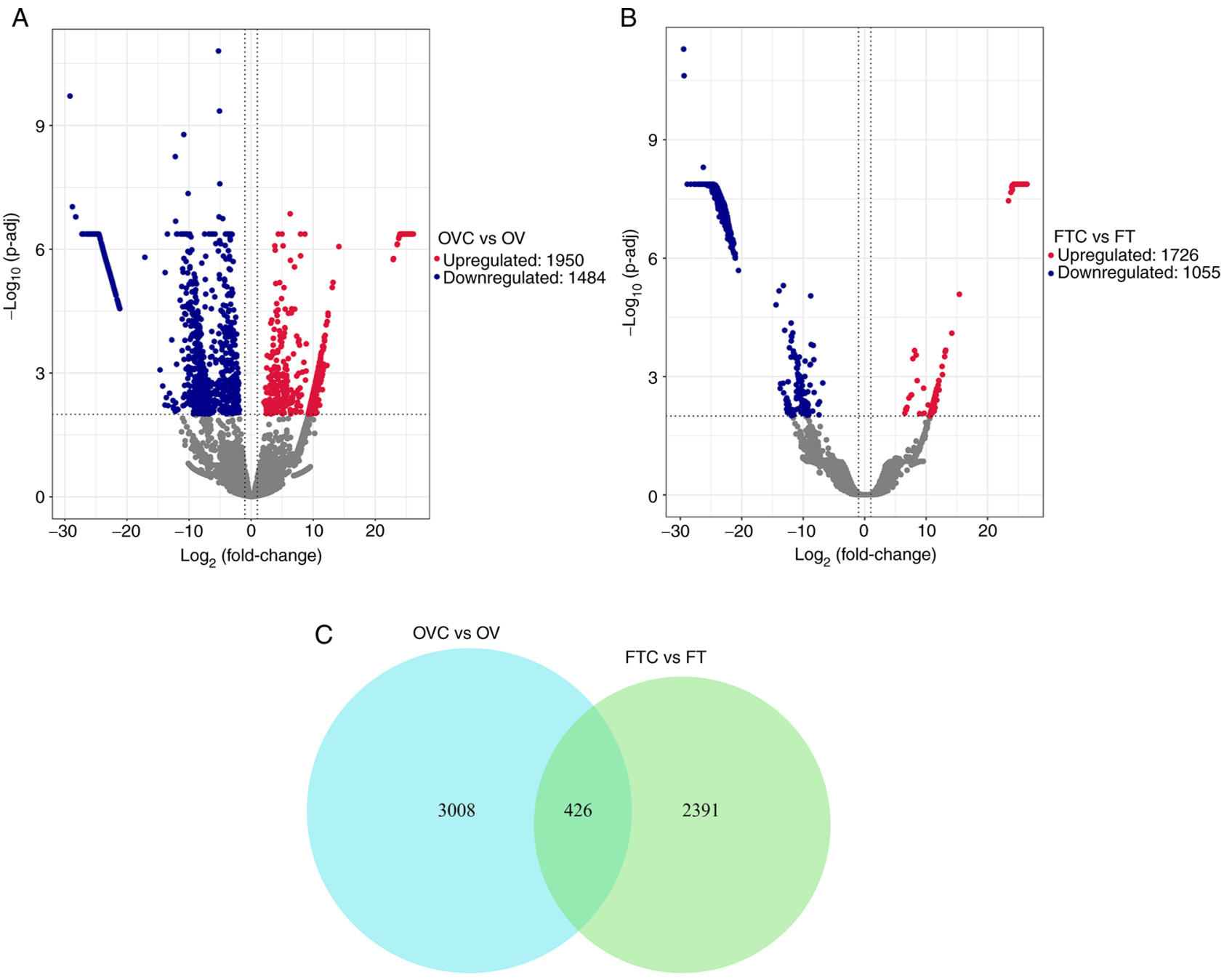

Figure 2. DEGs between healthy and cancerous ovarian and fallopian tissues. Volcano plots indicating the upregulated (red dots) and downregulated (blue dots) DEGs between (A) FTC and FT groups and (B) OVC and OV groups. The threshold for these data are q-value $<0.001$ and $\log 2$ (fold-change) $>1$. (C) Number of overlapping DEGs between cancerous and healthy tissues. DEGs, differentially expressed genes; FT, healthy fallopian tube; FTC, fallopian tube cancer; OV, healthy ovarian tissue; OVC, ovarian cancer; $\mathrm{p}$-adj, adjusted P-value.

$1.16 \%)$ and 'transcriptional misregulation in cancer' $(246$ unigenes to 125 koIDs; $1.16 \%$ ). The catalog of the identified unigenes provided a broad gene transcription profile of $M$. fortis and a foundation for the screening of DEGS to reveal the underlying mechanisms of primary OVC in M. fortis.

DEGs involved in M. fortis primary OVC. Identification and characterization of DEGs derived from comparing FTC with FT and OVC with OV group were crucial for revealing the underlying mechanisms of primary OVC. To investigate the DEGs, RNA-seq technology was used. Clean reads from the four M. fortis tissue groups were mapped to the de novo assembly transcriptome reference sequences of $M$. fortis using Bowtie2 (version 2.4.1, http://bowtie-bio.sourceforge. net/bowtie2/index.shtml) (28). The expression levels of unigenes were calculated using the RSEM algorithm (29). In the present study, bioinformatics analysis identified 3,434 DEGs between the OVC and the OV groups (Table SVII), including 1,950 significantly upregulated and 1,484 significantly downregulated genes (Fig. 2A). In the comparison between the FTC and the FT group, 2,817 DEGs were identified (Table SVIII), including 1,762 significantly upregulated and 1,055 significantly downregulated genes (Fig. 2B). The number of overlapping DEGs between fallopian tube tissues and ovarian tissues was 426 (Fig. 2C).

In the OVC vs. OV group, there were seven upregulated expression genes, containing TNNC1, RAP1B,RAC1,HA12,BTG3,CYR61 and RHOA, while only one downregulated gene, P53. Among the seven upregulated expression genes, five significantly upregulated DEGs were identified that were related to OVC cell proliferation and migration: CYR61, RAP1B, RHOA, RAC1 and BTG3. In the FTC vs. FT group, two significantly upregulated DEGs, troponin $\mathrm{C}$ (TNNC1) and hyaluronan dodecasaccharides (HA12) were identified (Fig. 3). Overexpression of TNNC1 may be beneficial for cell transfer (30) and high expression of HA12 may promote endothelial cell morphogenesis (31).

KEGG functional enrichment analysis of DEGs. Investigating the statistically enriched KEGG pathways related to the DEGs revealed that the most significant and most frequently enriched terms for upregulated unigenes in the OVC compared with the 

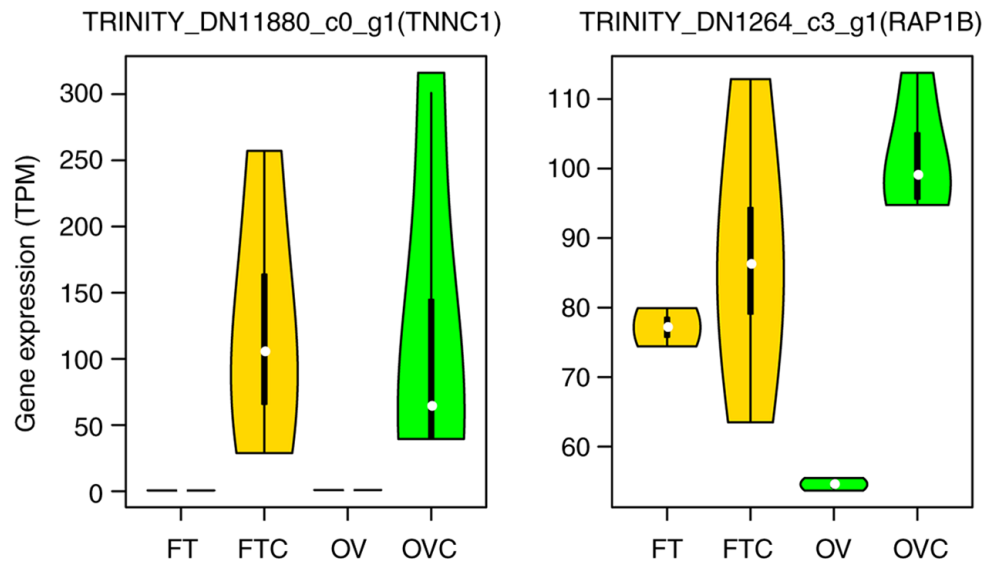

TRINITY_DN1451_C0_g1(RAC1)
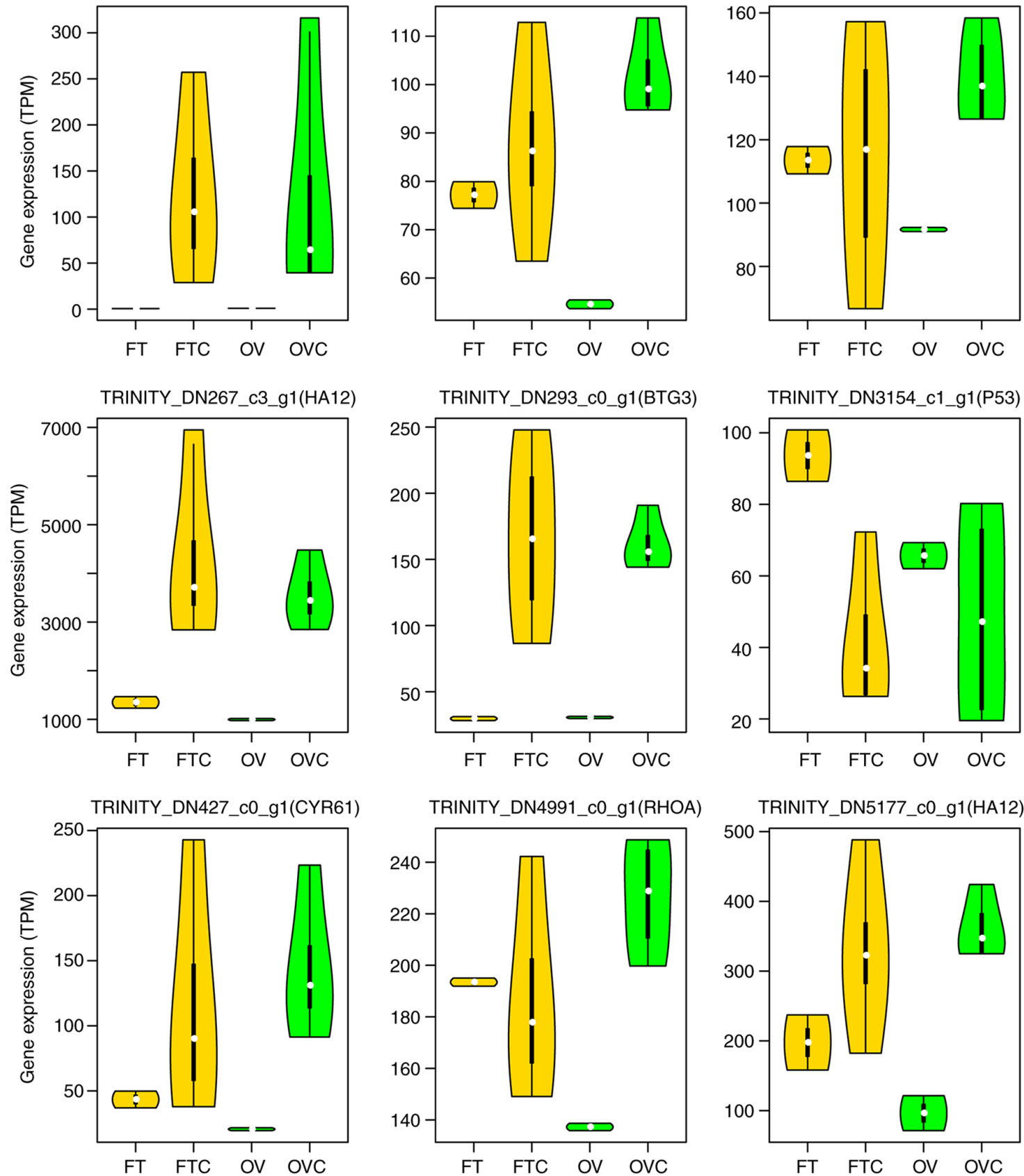

TRINITY_DN4991_c0_g1(RHOA)
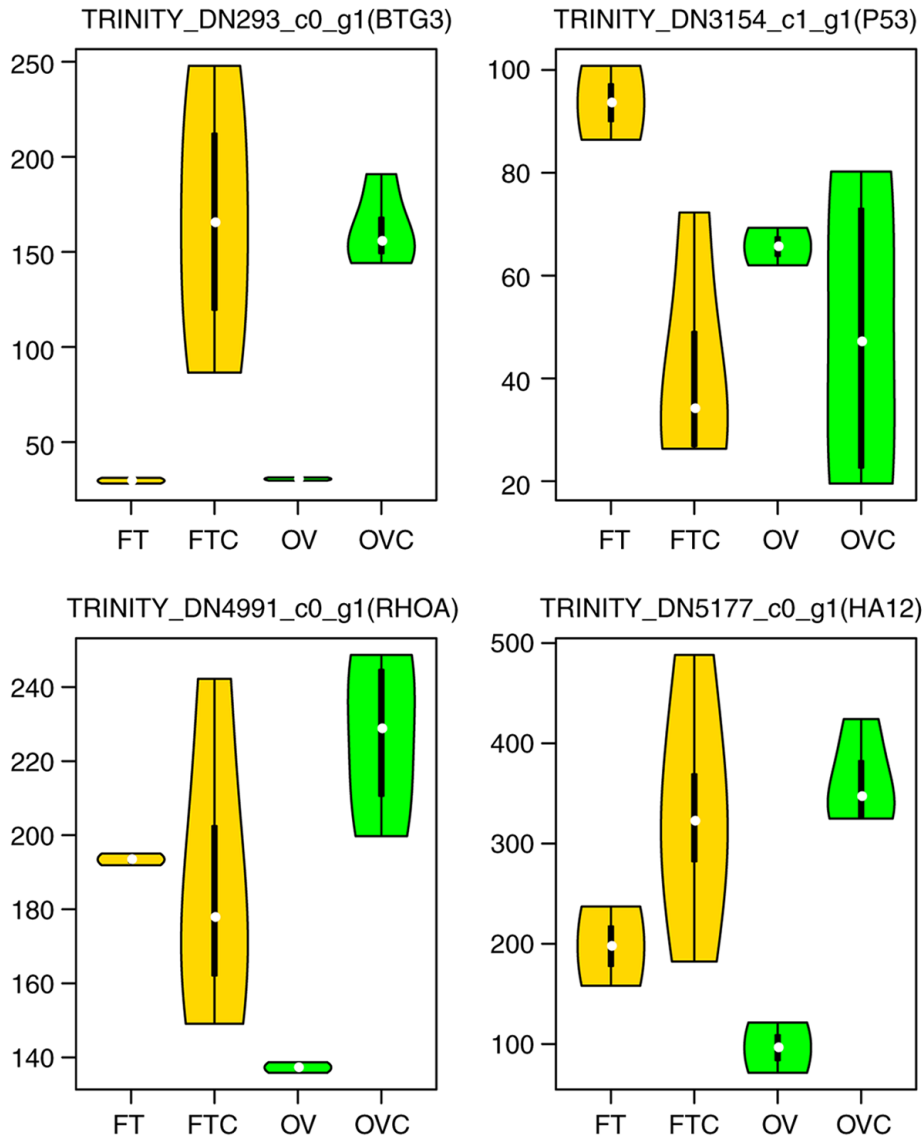

TRINITY_DN6231_c1_g1(BTG3)

TRINITY_DN7645_C1_g1(HA12)
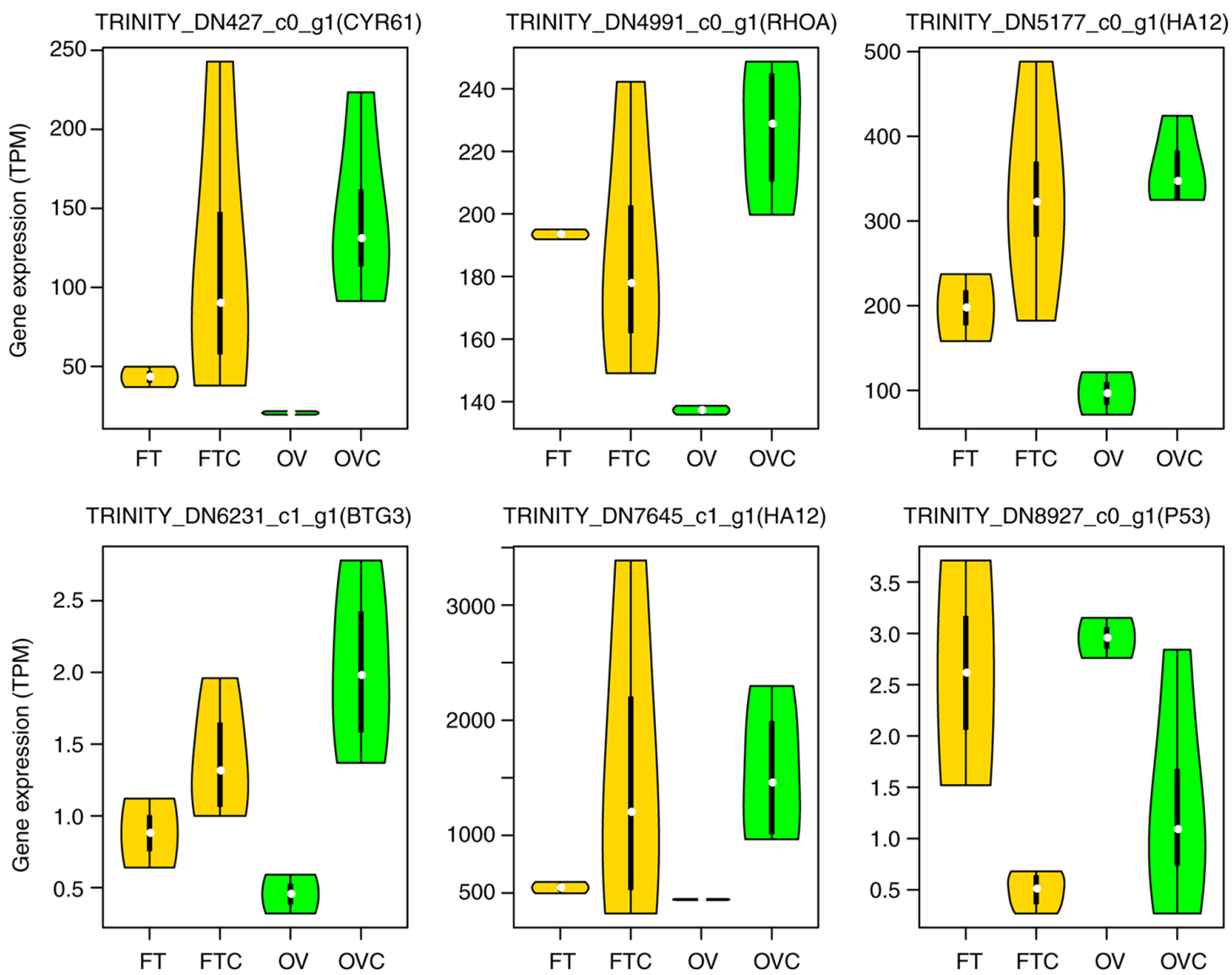

TRINITY_DN8927_C0_g1(P53)

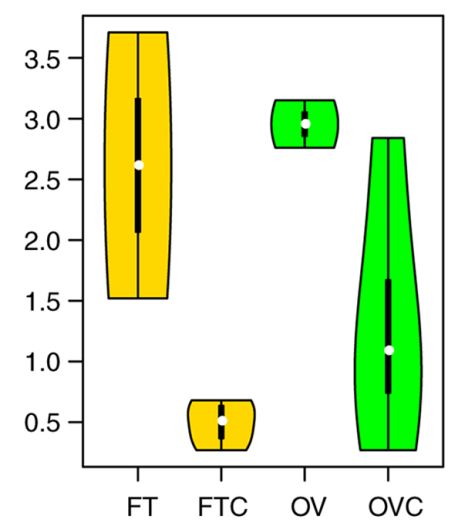

Figure 3. Violin plots of DEGs related to cancer development. DEGs analyzed include CYR61, RAP1B, RHOA, RAC1, BTG3, TNNC1, HA12 and p53 (different transcripts could be annotated with the same gene symbol). BTG3, BTG antiproliferation factor 3; CYR61, cysteine-rich angiogenic inducer 61; DEGs, differentially expressed genes; FT, healthy fallopian tubes; FTC, fallopian tube cancer; HA12, hyaluronan dodecasaccharides; RAP1B, Ras-related protein Rap-1b; RHOA, Ras homolog family member A; OV, healthy ovarian tissue; OVC, ovarian cancer; TNNC1, troponin C. 
A

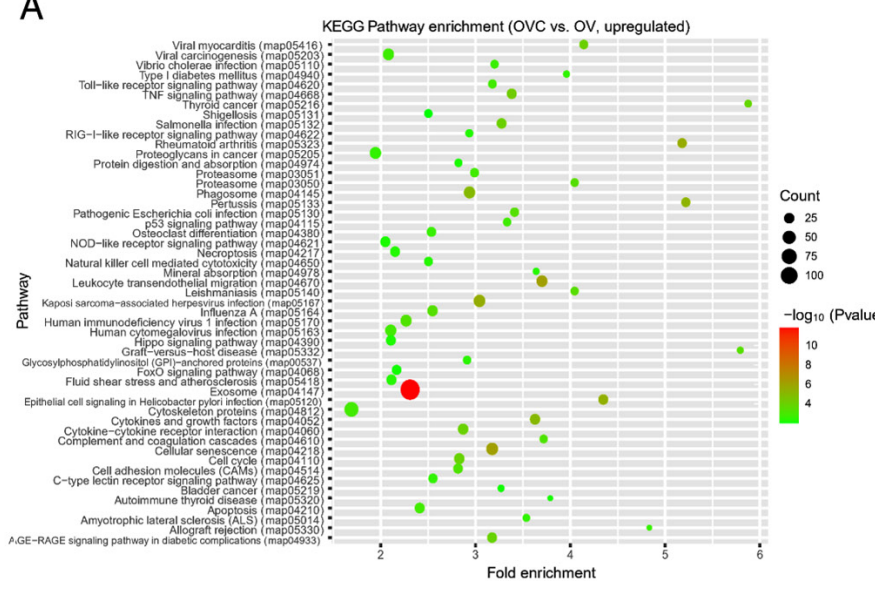

C

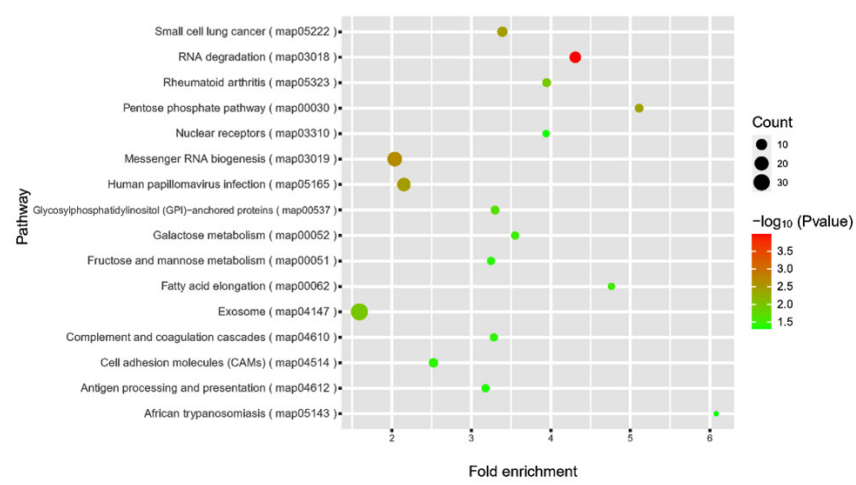

B

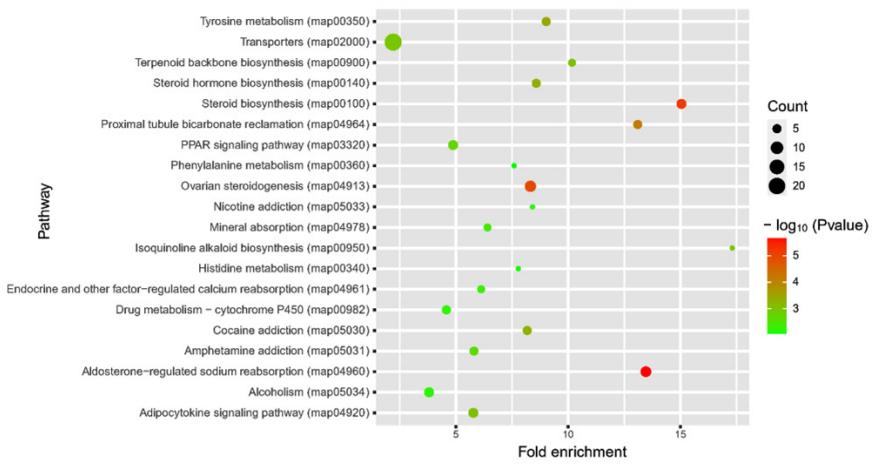

DEGG Pathway enrichment (FTC vs. FT, downregulated)

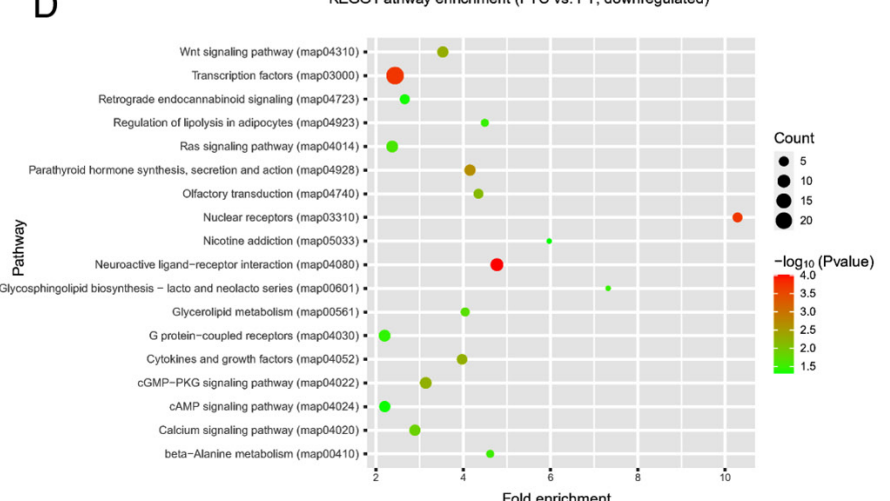

Figure 4. KEGG enrichment analysis of DEGs. (A) KEGG enrichment analysis of upregulated DEGs between OVC and OV groups. (B) KEGG enrichment analysis of downregulated DEGs between OVC and OV groups. (C) KEGG enrichment analysis of upregulated DEGs between FTC and FT groups. (D) KEGG enrichment analysis of downregulated DEGs between FTC and FT groups. AGE, advanced glycation end products; cGMP, cyclic guanosine monophosphate; DEGs, differentially expressed genes; FT, healthy fallopian tubes; FTC, fallopian tube cancer; GTP, guanosine triphosphate; KEGG, Kyoto Encyclopedia of Genes and Genomes; NOD, nucleotide-binding oligomerization domain; OV, healthy ovarian tissue; OVC, ovarian cancer; PKG, protein kinase G; PPAR, peroxisome proliferator-activated receptor; RAGE, receptor for advanced glycation end products.

OV group were signaling pathway, cell growth and proliferation-associated terms, including 'cytoskeleton proteins', 'cellular senescence', 'cell cycle', 'cytokines and growth factors', 'exosome', 'cytokine-cytokine receptor interaction', 'cell adhesion molecules (CAMs)', 'hippo signaling pathway' and 'TNF signaling pathway' (Fig. 4A; Table SIX). Moreover, the downregulated DEGs in the OVC compared with the OV group were enriched in steroid biosynthesis-related terms, including 'steroid biosynthesis', 'steroid hormone biosynthesis' and 'ovarian steroidogenesis' (Fig. 4B; Table SX). In the FTC vs. FT group comparisons, upregulated DEGs were mainly enriched in the terms 'exosome' and 'messenger RNA biogenesis' (Fig. 4C; Table SXI), whereas downregulated DEGs were mainly enriched in the terms 'Ras signaling pathway', 'cGMP-PKG signaling pathway', 'G protein-coupled receptors', 'calcium signaling pathway', 'cAMP signaling pathway' and 'Wnt signaling pathway' (Fig. 4D; Table SXII).

\section{Discussion}

The pathogenesis of OVC is complicated and, therefore, improved animal models are required to investigate the underlying mechanisms of OVC. Nowadays, more induced and less spontaneous models of small animals were used for disease researches, however spontaneous cancer model similar with human metabolism can offer more translatable results (32). In the present study, four $M$. fortis females with spontaneous OVC were identified, some of which were hereditary. The cancerous individuals displayed ascites and histopathological $\mathrm{H} \& \mathrm{E}$ staining demonstrated the presence of papillary cancer cell structures in the ovarian tissues. Spontaneous cancer models are valuable and understanding the pathogenesis of spontaneous OVC in M.fortis at the genetic level is of great importance to the stabilization of this spontaneous ovarian cancer animal model.

During the DEG analysis, biomarkers related to the development of cancer were identified. p53, located in the chromosome 17 (17p13.1) and known as cancer suppressor gene, can determine the degree of DNA variation (33). If the variation is small, cell regeneration may be promoted; if the variation is large, apoptosis may be induced. Furthermore, the induction of apoptosis is closely related to cell proliferation and differentiation, with $50 \%$ of human tumors possessing a mutation in p53 (33). However, CYR61 can reduce p53 promoter activity, thereby reducing the expression level of p53 (34). In the present study, the expression of p53 (TRINITY_DN3154 c1_g1 and TRINITY_DN8927_c0_g1) in cancerous tissues (both OVC and FTC) was also lower compared with normal 
tissues. This suggested that in OVC tissues, CYR61 may increase cell proliferation by inhibiting the expression of p53.

Tumor invasion and metastasis, which are both hallmarks of tumor malignancy (35), frequently coincide with the loss of E-cadherin-mediated cell-cell adhesion (36). Previous studies have shown that the overexpression of RHOA (37), RAP1B (38) and RAC1 (39) is beneficial for tumor invasion. In the present study, RAP1B, RHOA and RAC1, were markedly upregulated in the OVC group compared with the OV group, which indicated that these genes may serve a key role in promoting cell metastasis in OVC tissues. Previous studies have reported that BTG3 can inhibit cell proliferation and metastasis, therefore affecting cancer development (40-42). However, it has been demonstrated that mRNA expression levels of transducer of ERBB-2,1 (TOB1) and BTG2 are decreased in most types of cancer compared with healthy tissues, whereas BTG3 is upregulated (43); in the present study, the gene expression of BTG3 was upregulated in both OVC and FTC tissues. Further analysis of CYR61, RAP1B, RHOA, RAC1 and BTG3 may help reveal the underlying mechanisms of primary OVC.

As $M$. fortis does not yet have a completely sequenced genome, the present study employed de novo RNA-seq technology to identify DEGs. An unclear exon and intron structure and the poor preservation state of the residual RNA led to a lack of molecular biology verification experiments for the identified DEGs. In future work, following the ongoing study of the de novo assembly of the M. fortis genome, molecular biology verification experiments will be performed and molecular breeding technology (using DNA markers that are tightly linked to phenotypic traits to assist in a selection scheme for a particular breeding objective) will be employed to build a stable natural OVC incidence model of $M$. fortis.

In summary, to the best of our knowledge, the present study was the first to characterize the $M$. fortis de novo transcriptome and to perform RNA-seq analysis to determine the genetic differences between OVC, OV, FTC and FT animal groups. The results demonstrated that the enriched biological pathways differed between cancerous and healthy tissues. Future analysis of these pathways will help to further reveal the pathogenesis of primary OVC in M.fortis. The some DEGs in this study also were reported as marker genes associated with human OVC, such as CYR61 (44), RAP1B (45), RHOA (46), RAC1 (47) and BTG3 (48). Hence, $M$. fortis could be used to better understand human OVC.

\section{Acknowledgements}

Not applicable.

\section{Funding}

The present study was supported by The Natural Science Foundation of Hunan Province (grant no. 2020JJ4110) and The Science and Technology Planning Project of Changsha (grant no. kq1801070).

\section{Availability of data and materials}

All raw sequencing data has been deposited in the National Centre for Biotechnology Information Sequence Read Archive
(BioProject identifier, PRJNA687349; SRA accessions, SRR13329310-SRR13329323).

\section{Authors' contributions}

ZZ and QH designed the experiments. QL, WZ, JW, BL and $\mathrm{SH}$ performed the experiments and sample collection. $\mathrm{QH}$, DZ and MG conducted the bioinformatics analysis. QH, DZ and BL confirm the authenticity of all the data. MG drafted the work manuscript. $\mathrm{BL}, \mathrm{QH}$ and $\mathrm{ZZ}$ revised the manuscript critically for important intellectual content. All authors read and approved the final manuscript.

\section{Ethics approval and consent to participate}

This study was performed in strict accordance with the recommendations of the Laboratory of Animal Welfare \& Ethics Committee of China. The protocol was approved by the Laboratory Animal Welfare and Ethics Committee of Central South University (Changsha, China; approval no. 2018sydw0236).

\section{Patient consent for publication}

Not applicable.

\section{Competing interests}

The authors declare that they have no competing interests.

\section{References}

1. Chen W, Zheng R, Baade PD, Zhang S, Zeng H, Bray F, Jemal A, $\mathrm{Yu}$ XQ and He J: Cancer statistics in China, 2015. CA Cancer J Clin 66: 115-132, 2016.

2. Ishizuka B: Current Understanding of the etiology, symptomatology, and treatment options in premature ovarian insufficiency (POI). Front Endocrinol (Lausanne) 12: 626924, 2021.

3. Chen SN, Chang R, Lin LT, Chern CU, Tsai HW, Wen ZH, Li YH, Li CJ and Tsui KH: MicroRNA in ovarian cancer: Biology, pathogenesis, and therapeutic opportunities. Int J Environ Res Public Health 16: 1510, 2019.

4. Stewart C, Ralyea C and Lockwood S: Ovarian cancer: An integrated review. Semin Oncol Nurs 35: 151-156, 2019.

5. Lutgendorf SK and Sood AK: Biobehavioral factors and cancer progression: Physiological pathways and mechanisms. Psychosom Med 73: 724-730, 2011.

6. Bigorie V, Morice P, Duvillard P, Antoine M, Cortez A, Flejou JF, Uzan S, Darai E and Barranger E: Ovarian metastases from breast cancer: Report of 29 cases. Cancer 116: 799-804, 2010.

7. Mikula-Pietrasik J, Uruski P, Tykarski A and Książek K: The peritoneal 'soil' for a cancerous 'seed': A comprehensive review of the pathogenesis of intraperitoneal cancer metastases. Cell Mol Life Sci 75: 509-525, 2018.

8. Parashar D, Nair B, Geethadevi A, George J, Nair A, Tsaih SW, Kadamberi IP, Gopinadhan Nair GK, Lu Y, Ramchandran R, et al: Peritoneal spread of ovarian cancer harbors therapeutic vulnerabilities regulated by FOXM1 and EGFR/ERBB2 signaling. Cancer Res 80: 5554-5568, 2020.

9. Levy A, Medjhoul A, Caramella C, Zareski E, Berges O, Chargari C, Boulet B, Bidault F, Dromain C and Balleyguier C: Interest of diffusion-weighted echo-planar MR imaging and apparent diffusion coefficient mapping in gynecological malignancies: A review. J Magn Reson Imaging 33: 1020-1027, 2011.

10. Li Z, Zheng W, Wang H, Cheng Y, Fang Y, Wu F, Sun G, Sun G, Lv C and Hui B: Application of animal models in cancer research: Recent progress and future prospects. Cancer Manag Res 13: 2455-2475, 2021. 
11. Overgaard NH, Fan TM, Schachtschneider KM, Principe DR, Schook LB and Jungersen G: Of Mice, Dogs, Pigs, and Men: Choosing the appropriate model for immuno-oncology research. ILAR J 59: 247-262, 2018.

12. Jayson GC, Kohn EC, Kitchener HC and Ledermann JA: Ovarian cancer. Lancet 384: 1376-1388, 2014.

13. Katt ME, Placone AL, Wong AD, Xu ZS and Searson PC: In Vitro tumor models: Advantages, disadvantages, variables, and selecting the right platform. Front Bioeng Biotechnol 4: 12, 2016

14. Kamdem JP, Duarte AE, Ibrahim M, Lukong KE, Barros LM and Roeder T: Bibliometric analysis of personalized humanized mouse and Drosophila models for effective combinational therapy in cancer patients. Biochim Biophys Acta Mol Basis Dis 1866: 165880, 2020.

15. Yu YJ, Su ZJ, Zhou ZJ and Ma YD: Pathological observation on spontaneous epithelial ovarian cancer in reed vole. Chin J V Sci: 1070-1073, 2008 (In Chinese).

16. Zhou ZJ, Yu YJ, Su ZJ and Tang LF: Expression of heat shock proteins HSP27, HSP70 and HSP90 in spontaneous ovarian cancer of Microtus fortis. Chin J Comp Med 4: 26-28, 2005 (In Chinese).

17. Yu Y: An investigation of pathological changes in Captive-bred Microtus fortis. Chin J Zool 38: 71-73, 2003 (In Chinese).

18. Zheng M, Hu Y, Gou R, Nie X, Li X, Liu J and Lin B: Identification three LncRNA prognostic signature of ovarian cancer based on genome-wide copy number variation. Biomed Pharmacother 124: 109810, 2020.

19. Hu Y, Xu Y, Lu W, Yuan Z, Quan H, Shen Y and Cao J: De novo assembly and transcriptome characterization: Novel insights into the natural resistance mechanisms of Microtus fortis against Schistosoma japonicum. BMC Genomics 15: 417, 2014

20. Marquardt N, Feja M, Hünigen H, Plendl J, Menken L, Fink H and Bert B: Euthanasia of laboratory mice: Are isoflurane and sevoflurane real alternatives to carbon dioxide? PLoS One 13: e0203793, 2018.

21. Laferriere CA, Leung VS and Pang DS: Evaluating intrahepatic and intraperitoneal sodium pentobarbital or ethanol for mouse euthanasia. J Am Assoc Lab Anim Sci 59: 264-268, 2020.

22. Bolger AM, Lohse M and Usadel B: Trimmomatic: A flexible trimmer for Illumina sequence data. Bioinformatics 30 2114-2120, 2014

23. Grabherr MG, Haas BJ, Yassour M, Levin JZ, Thompson DA, Amit I, Adiconis X, Fan L, Raychowdhury R, Zeng Q, et al: Full-length transcriptome assembly from RNA-Seq data without a reference genome. Nat Biotechnol 29: 644-652, 2011.

24. R Core Team: R: A Language and Environment for Statistical Computing. R Foundation for Statistical Computing, Vienna, Austria. Computing, 14: pp12-21, 2009.

25. Eddy SR: Profile hidden markov models. Bioinformatics 14 755-763, 1998

26. Almagro Armenteros JJ, Tsirigos KD, Sønderby CK, Petersen TN, Winther O, Brunak S, von Heijne G and Nielsen H: SignalP 5.0 improves signal peptide predictions using deep neural networks. Nat Biotechnol 37: 420-423, 2019

27. Love MI, Huber W and Anders S: Moderated estimation of fold change and dispersion for RNA-seq data with DESeq2. Genome Biol 15: 550, 2014

28. Langmead B and Salzberg SL: Fast gapped-read alignment with Bowtie 2. Nat Methods 9: 357-359, 2012.

29. Li B and Dewey CN: RSEM: Accurate transcript quantification from RNA-Seq data with or without a reference genome. BMC Bioinformatics 12: 323, 2011

30. Leung CS, Yeung TL, Yip KP, Pradeep S, Balasubramanian L, Liu J, Wong KK, Mangala LS, Armaiz-Pena GN, Lopez-Berestein G,etal:Calcium-dependentFAK/CREB/TNNC1 signalling mediates the effect of stromal MFAP5 on ovarian cancer metastatic potential. Nat Commun 5: 5092, 2014.

31. Takahashi Y, Li L, Kamiryo M, Asteriou T, Moustakas A, Yamashita Hand Heldin P: Hyaluronan fragments induce endothelial cell differentiation in a CD44- and CXCL1/GRO1-dependent manner. J Biol Chem 280: 24195-24204, 2005.

32. Onaciu A, Munteanu R, Munteanu VC, Gulei D, Raduly L, Feder RI, Pirlog R, Atanasov AG, Korban SS, Irimie A and Berindan-Neagoe I: Spontaneous and induced animal models for cancer research. Diagnostics (Basel) 10: 660, 2020.
33. Cicalese A, Bonizzi G, Pasi CE, Faretta M, Ronzoni S, Giulini B, Brisken C, Minucci S, Di Fiore PP and Pelicci PG: The tumor suppressor p53 regulates polarity of self-renewing divisions in mammary stem cells. Cell 138: 1083-1095, 2009.

34. Yue XT, Zhao Y, Xu Y, Zheng M, Feng Z and Hu W: Mutant p53 in Cancer: Accumulation, Gain-of-function, and therapy. J Mol Biol 429: 1595-1606, 2017.

35. Barkal AA, Brewer RE, Markovic M, Kowarsky M, Barkal SA Zaro BW, Krishnan V, Hatakeyama J, Dorigo O, Barkal LJ and Weissman IL: CD24 signalling through macrophage Siglec-10 is a target for cancer immunotherapy. Nature 572: 392-396, 2019.

36. Lee KB, Byun HJ, Park SH, Park CY, Lee SH and Rho SB: CYR61 controls $\mathrm{p} 53$ and $\mathrm{NF}-\mathrm{\kappa B}$ expression through PI3K/Akt/mTOR pathways in carboplatin-induced ovarian cancer cells. Cancer Lett 315: 86-95, 2012.

37. von Karstedt S, Conti A, Nobis M, Montinaro A, Hartwig T, Lemke J, Legler K, Annewanter F, Campbell AD, Taraborrelli L, et al: Cancer cell-autonomous TRAIL-R signaling promotes KRAS-driven cancer progression, invasion, and metastasis. Cancer Cell 27: 561-573, 2015.

38. Lin KT, Yeh YM, Chuang CM, Yang SY, Chang JW, Sun SP, Wang YS, Chao KC and Wang LH: Glucocorticoids mediate induction of microRNA-708 to suppress ovarian cancer metastasis through targeting Rap1B. Nat Commun 6: 5917, 2015.

39. Leng R, Liao G, Wang H, Kuang J and Tang L: Rac1 expression in epithelial ovarian cancer: Effect on cell EMT and clinical outcome. Med Oncol 32: 329, 2015.

40. Ren XL, Zhu XH, Li XM, Li YL, Wang JM, Wu PX, Lv ZB, Ma WH, Liao WT, Wang W, et al: Down-regulation of BTG3 promotes cell proliferation, migration and invasion and predicts survival in gastric cancer. J Cancer Res Clin Oncol 141: 397-405, 2015.

41. Mao D, Qiao L, Lu H and Feng Y: B-cell translocation gene 3 overexpression inhibits proliferation and invasion of colorectal cancer SW480 cells via Wnt/ $\beta$-catenin signaling pathway. Neoplasma 63: 705-716, 2016.

42. Deng B, Zhao Y, Gou W, Chen S, Mao X, Takano Y and Zheng H: Decreased expression of BTG3 was linked to carcinogenesis, aggressiveness, and prognosis of ovarian carcinoma. Tumor Biol 34: 2617-2624, 2013.

43. Bai Y, Qiao L, Xie N, Shi Y, Liu N and Wang J: Expression and prognosis analyses of the Tob/BTG antiproliferative (APRO) protein family in human cancers. PLoS One 12: e0184902, 2017.

44. Liu T, Yang Y, Xie Z, Luo Q, Yang D, Liu X, Zhao H, Wei Q, Liu Y, Li L, et al: The RNA binding protein QKI5 suppresses ovarian cancer via downregulating transcriptional coactivator TAZ. Mol Ther Nucleic Acids 26: 388-400, 2021.

45. Cui G, Wang C, Lin Z, Feng X, Wei M, Miao Z, Sun Z and Wei F: Prognostic and immunological role of Ras-related protein Raplb in pan-cancer. Bioengineered 12: 4828-4840, 2021.

46. Wei X, Lou H, Zhou D, Jia Y, Li H, Huang Q, Ma J, Yang Z, Sun C, Meng Y, et al: TAGLN mediated stiffness-regulated ovarian cancer progression via RhoA/ROCK pathway. J Exp Clin Cancer Res 40: 292, 2021.

47. Wang X, Wei Z, Tang Z, Xue C, Yu H, Zhang D, Li Y, Liu X, Shi Y, Zhang L, et al: IL-37b $\Delta 1-45$ suppresses the migration and invasion of endometrial cancer cells by targeting the Rac1/NF-кB/MMP2 signal pathway. Lab Invest 101: 760-774, 2021.

48. Yanagida S, Taniue K, Sugimasa H, Nasu E, Takeda Y, Kobayashi M, Yamamoto T, Okamoto A and Akiyama T: ASBEL, an ANA/BTG3 antisense transcript required for tumorigenicity of ovarian carcinoma. Sci Rep 3: 1305, 2013.

This work is licensed under a Creative Commons Attribution-NonCommercial-NoDerivatives 4.0 International (CC BY-NC-ND 4.0) License. 